\title{
Impact of neurotherapy treatment on knee pain
}

\author{
Ajay Gandhi*, Lakhveer Singh, Mayank Pandey \\ Lajpatrai Mehra Neurotherapy Research and Training Institute Mohali, Punjab, India
}

\section{KEY WORDS}

Neurotherapy

Knee pain

Adrenal gland

*Corresponding Author:

Ajay Gandhi

Lajpatrai Mehra Neurotherapy

Research and Training Institute Mohali

Punjab, India

Contact no: 9815220699

E-mail: Imntajay@gmail.com

\section{Introduction}

Knee pain is most common in elderly population especially among women who are more prone to knee pain (1). Physical therapy exercises, analgesics, knee replacement surgery, and weight reduction in people who are overweight are considered as some of the effective interventions for reducing knee pain $(2,3,4)$. About $25 \%$ of people over the age of 50 experience knee pain and surgery does not provide significant improvement to pain and function of knees (4). Acupuncture is also considered as an effective therapy for knee pain (5). Here in this study Neurotherapy is used as a effective treatment for reducing knee pain significantly. The human body contains different organs and glands which have specific functions to do. Each releases certain chemicals and hormones in specified quantity which takes care of bodily activities e.g. Mouth secretes saliva which helps in digestion, intestines absorbs the nutrients from digested food etc. The assumption of the therapy is that when each organ/gland is functioning normally and releasing its secretions properly, then body remains in a healthy and balanced state. If these organs/glands are not working properly and their secretions are impaired (either increased or decreased) then it leads to disorder or disease.

Neurotherapy deals with the whole body/mind system in. Body can heal itself by generating the requisite hormones and chemicals. The philosophy behind Neurotherapy involves "activating or deactivating the organ(s), through pressure or massage on the nerve channels in order to stimulate or depress the blood, \& other body fluids and the neurotransmission enbles homeostasis in the body thus helping it to regain its equilibrium. Neurotherapy claims to focus at the cause not at the symptoms of the disease. These centers are distributed throughout the India as it showed positive impact on the patient, Neurotherapy can be considered as a effective way to treat knee pain an as adjunct or alternative therapy for which more studies are required.

\section{Case presentation}

A female patient, aged 65 was suffering from knee pain from last six years and was also taking some medicines (Ayurveda and Allopathic) for the same problem. But these treatments were not giving any considerable results and pain was still same. When the patient got to know about Neurotherapy Center at Mohali, Chandigarh after attending a camp at sector 70 organized by authors, she sought treatment at camp and felt better. Therefore, she came to the center for further treatment. Author (Neurotherapist) gave her treatment for 82 sittings at Neurotherapy centre, Mohali. Treatment was given by three different Neurotherapists working at the centre by following same protocol. She was treated with (6) ADR/(8) ADR: This treatment applied on the thoracic region of the spine from $4^{\text {th }} \mathrm{V}$ to $12^{\text {th }} \mathrm{V}$. It is believed to affect the adrenal gland and produces a positive effect to reduce the pain. The adrenal medulla is stimulated by sympathetic nervous system through preganglionic fibers originating from T5-T11 vertebrae in Thoracic Spinal Cord (6). Glucocorticoids (GCs) are stimulated with this treatment. It helps in treatment of inflammatory disorders.

She stopped taking medicines on her own after 8 sittings of Neurotherapy treatment and continued Neurotherapy as singular treatment for knee pain.

\section{Hypothetical mechanism of action}

It is believed that various acusitive (pressure) points that there are in the human body. When these points are pressed certain glands and organs are stimulated which release the respective hormones and other Chemicals. In this case, massage and pressing was carried out to stimulate adrenal gland. As Neurotherapist (the author) is an expert of Neurotherapy, he claims to easily recognize these different pressure points.

During stress condition adrenal cortex synthesize and release GC. The stimulation of adrenal gland was Carried out by applying pressure besides the T6-T12 region of spine. The 
main function of GC hormone is to maintain and restore the basal and stress-related body homeostasis. This protective action of GCs is hypothesised to be accomplished by its metabolic, cardio protective, and anti-inflammatory actions. GCs are known to enhance plasma glucose levels and promote an increased cardiac output and high blood pressure, while protecting against excessive inflammation therefore by increasing the blood flow to the weaker region of body, impaired parts of the body can be treated. Although we are not able to present the biochemical investigation before and after treatment, We speculate that the rescue from pain may be governed by various transposant that can be measured before, during and after the treatment. We will be presenting these in the next case report and encourage other to undertake the research activity.

\section{Diagnosis}

After 82 sittings ( 6 days per week), patient was satisfied with the results. From last 6 years she was unable to sit with her legs crossed, to climb stairs. However, after Neurotherapy treatment she was relieved in a short period of time. As she stopped taking medicines only after attending 8 sittings of Neurotherapy (15 minutes/sitting), Neurotherapy may be responsible for reducing knee pain that she was experiencing from last 6 years. She was unaffected by medicines she was taking.

\section{Limitation}

As pain detection test is expensive and patient was unable to afford this test, therefore, the observations are on the basis of patient's experience further research is required in the field.

\section{Conclusion}

A case of $65 \mathrm{y} / \mathrm{o}$ female patient suffering from knee pain from last 6 years reduced by Neurotherapy intervention.

\section{Acknowledgement}

I want to thank my whole Neruotherapy team for their support and to Pooja and Priya Mehra who contributed to complete this case report successfully.

\section{Informed consent}

Yes report has been written from the center to which authors are affiliated.

\section{Conflict of interest}

The Authors declare no conflict of interest.

\section{Source of funding}

\section{Nil}

Received Date: 09-02-20; Revised Date: 11-02-20

Accepted Date: $12-02-20$

\section{References}

1. McAlindon TE, Cooper C, Kirwan JR, Dieppe PA. Knee pain and disability in the community. Rheumatology. 1992 Mar 1;31(3):189-92.

2. Lack S, Neal B, De Oliveira Silva D, Barton C. "How to manage patellofemoral pain - Understanding the multifactorial nature and treatment options". Physical Therapy in Sport. 2018 Jul; 32:155-166.

3. Knee reviver https://www.pontesmedical.com/en/portfolio/kneereviver/

4. Siemieniuk RA, Harris IA, Agoritsas T, Poolman RW, Brignardello-Petersen R, Van de Velde S, Buchbinder R, Englund M, Lytvyn L, Quinlan C, Helsingen L. Arthroscopic surgery for degenerative knee arthritis and meniscal tears: a clinical practice guideline. Bmj. 2017 May 10; 357:j1982.

5. Zhang Q Yue J, Golianu B, Sun Z, Lu Y. "Updated systematic review and meta-analysis of acupuncture for chronic knee pain". Acupuncture in Medicine. 2017 Dec; 35(6):392-403. doi:10.1136/acupmed2016-011306

6. Siegel A, Sapru HN. Essential neuroscience. Lippincott Williams \& Wilkins; 2006. 